\title{
Visualización mediática de la ciencia: tipología de la infografía científica de prensa
}

\author{
Ma. Luisa Montes-Rojas *; Juanjo García-Gil **; David Alonso Leija-Román * \\ * Universidad Autónoma de Tamaulipas, Facultad de Arquitectura, Diseño y Urbanismo. México \\ Correo-e: mImontes@docentes.uat.edu.mx | ORCID iD: https://orcid.org/ 0000-0003-3138-1582 \\ Correo-e: dleija@docentes.uat.edu.mx | ORCID iD: https://orcid.org/0000-0001-5782-2767 \\ ** Universidad Ramon Llull, Facultad de Ciencias de la Salud Blanquerna. Barcelona \\ Correo-e: juanjosegg2@blanquerna.url.edu | ORCID iD: https://orcid.org/0000-0002-1448-3002
}

Recibido: 21-01-2019; 2a versión: 08-07-2019; Aceptado: 28-08-2019.

Cómo citar este artículo/Citation: Montes-Rojas, M. L.; García-Gil. J.; Leija-Román, D. A. (2020). Visualización mediática de la ciencia: tipología de la infografía científica de prensa. Revista Española de Documentación Científica, 43 (2), e266. https://doi. org/10.3989/redc.2020.2.1643

Resumen: Este artículo explora el tratamiento informativo de la infografía de la prensa internacional para emprender de manera eficaz procesos de comunicación y divulgación de los hechos relativos a la Ciencia y Tecnología (CyT). La integración de un método de análisis de contenido, aplicado a una muestra seleccionada de mejores prácticas en materia de infografía de periódicos y revistas que han recibido reconocimiento internacional a través de los premios Malofiej $(\mathrm{N}=149)$, fueron codificadas y analizadas para identificar características y patrones detallados con base a tres ejes de estudio: el planteamiento informativo (¿qué se dice?), los objetos gráficos y su correspondencia (¿cómo se dice?) y la función que desempeñan los infográficos (¿para qué se dice?). Los resultados indican que estas piezas informativas emplean distintos planteamientos informativos que reflejan en sus mensajes, los cuales conllevan al uso particular de determinados recursos de expresión (objetos gráficos), desempeñando de manera conjunta, diversas funciones comunicativas por parte del emisor con respecto al público al que se dirige; lo anterior, llevó a la conformación y descripción de tres modelos de infografía científica de prensa.

Palabras clave: Periodismo científico; infografía; alfabetización mediática; diseño de información; divulgación científica; visualización de datos.

\section{Media visualization of science: typology of scientific press infographics}

Abstract: This article explores the informative treatment of the infographics of the international press to undertake the communication and dissemination processes of facts related to Science and Technology (S\&T) in an efficient way. The integration of a content analysis method, applied to a selected sample of best practices in the field of infographics from newspapers and magazines, that have received international recognition through the Malofiej awards $(N=149)$, were analyzed and coded in order to identify characteristics and detailed patterns based on three axes of study: the informative approach (what is said?), the graphic objects and their correspondence (how do you say?) and the role played by the infographics (what is it for?). The results indicate that these informative pieces use different informative approaches, that reflect in their messages, which lead to the particular use of certain expression resources (graphic objects), jointly performing diverse communication functions on the part of the issuer with respect to the public to which is directed. All the above led to the formation and description of three scientific models for press infographics.

Keywords: Science Journalism; infographic; media literacy; information design; scientific dissemination; data visualization.

Copyright: ( 2020 CSIC. Este es un artículo de acceso abierto distribuido bajo los términos de la licencia de uso y distribución Creative Commons Reconocimiento 4.0 Internacional (CC BY 4.0). 


\section{INTRODUCCIÓN}

El incremento de datos e información, así como la presencia dominante de la comunicación visual, han generado que en la última década la prensa internacional se haya visto en la necesidad de aumentar el uso de la infografía para transferir de forma eficiente altas cantidades de información, tanto cualitativa como cuantitativa (Dur, 2012; Cairo, 2017). En este sentido, la prensa ha adoptado ciertos mecanismos que intentan facilitar la memoria y comprensión de la información (relaciones, rastreo, patrones, etc.), agilizando los procesos cognitivos de los lectores a través de herramientas visuales como mapas conceptuales, organizadores gráficos, diagramas de flujo, gráficos estadísticos, imágenes sintéticas, esquemas, etc. (Ware, 2012).

Es este escenario, la representación y diseño de información en los medios afronta grandes retos profesionales, propios del periodismo contemporáneo, debido a los nuevos formatos que se han gestado con la evolución de los medios (digitales e interactivos); las transformaciones que han ocurrido en los procesos de trabajo en las redacciones de prensa (Cairo, 2017); y las innovaciones tecnológicas que han surgido en materia de representación y visualización de imágenes y datos (nuevas gráficas y recursos visuales), que han llevado a que muchos profesionales se cuestionen si son los medios adecuados para hacer más comprensible la información.

La infografía como herramienta del periodismo especializado se ha convertido en los últimos años en un instrumento recurrente y clave para presentar información a través de historias, haciendo «uso de representaciones gráficas para ampliar la cognición, de esa forma, los términos visualización e infografía, dan nombre a una única disciplina» (Cairo, 2011). Asimismo, los fundamentos teóricos que sustentan hoy en día la visualización e infografía se han extendido a otras disciplinas relacionadas con la psicología cognitiva, la ergonomía y la psicología ambiental, proporcionando «los cimientos teóricos para la comunicación visual, el diseño gráfico, el diseño de interacción y la redacción técnica de textos que, combinados, definen el diseño de información» (Cairo, 2008).

La comunicación y divulgación de la CyT, por su parte, ha sido reflejo de estas nuevas tendencias y transformaciones en materia de diseño de información debido al incremento sustancial que se observa en la representación visual de datos de la ciencia, así como nuevas formas para modular, expresar y abordar la información tecnocientífica procedente de las principales áreas de estudio de la naturaleza: la física, la química, la biología, la geología y las matemáticas. Además de abordar nuevas especialidades emergentes como el periodismo sanitario, el medioambiental o el tecnológico, considerados como aplicaciones prácticas de las ciencias naturales en donde sus procedimientos, leyes generales, fundamentos y las dificultades para entender el lenguaje, son similares a los que informan sobre ciencias naturales (Elías, 2008).

De acuerdo con Elías (2008), el discurso de la Divulgación Mediática de la Ciencia y Tecnología (DMCYT) distingue tres principales planteamientos informativos: el divulgativo, donde se busca a la fuente (científicos divulgadores) para obtener el hallazgo científico necesario para construir el discurso, apoyado en la explicación de los fenómenos científicos (como son las tormentas solares) y narrados de forma intemporal sin involucrar los intereses de la población o relación alguna con el hecho noticioso; el periodístico, donde se analizan los asuntos que van más allá de la divulgación de la ciencia, como el uso de los recursos públicos destinados a la ciencia, el comportamiento ético y profesional de los científicos o la trascendencia de los resultados científicos; y el divulgativo periodístico, donde se unen ambos enfoques informativos que conllevan a la explicación de los conceptos científicos puros, al tiempo que, se contextualiza con criterios periodísticos (Elías, 2008).

Desde esta perspectiva, la eficacia de la infografía de prensa, especialmente cuando se abordan temas relativos a la Ciencia y Tecnología (CyT), ocurre a partir de la previa definición de un planteamiento informativo que puede ser divulgativo, periodístico o divulgativo periodístico (Elías, 2008), donde el discurso y los elementos gráficos (recursos de expresión) actúan en función de una intención comunicativa. Este artículo explora el tratamiento informativo de la infografía de la prensa internacional para emprender de manera eficaz procesos de comunicación y divulgación de los hechos relativos a la CyT. Las referencias de estudios similares a esta investigación son escasas, por lo que se considera pertinente contar con aportaciones orientadas hacia este objeto de estudio y que ofrezcan una visión actualizada del mismo.

Las investigaciones más recientes y cercanas a nuestro objeto de estudio se han centrado en estudiar el papel que desempeña la visualización e infografía y las representaciones visuales (fotografía, ilustración, caricatura, gráficos de datos, etc.) en la comprensión y difusión de temas relativos a la comunicación de la salud y el medio ambiente. Los resultados demuestran que los apoyos visuales en diversos segmentos de audiencias son una herramienta eficaz para aumentar la conciencia y el conocimiento en temas de riesgos de salud 
(véase Ancker y otros, 2006; Galesic y GarciaRetamero, 2011; Occa y Suggs, 2016; Stone y otros, 2017; Shanks y otros, 2017); además de mejorar la toma de decisiones, cambiar actitudes y reducir el comportamiento de riesgo (GarcíaRetamero y Cokely, 2013).

Otros estudios relativos a la comunicación de la ciencia y el medio ambiente demuestran que la visualización e infografía puede aumentar la participación de los individuos con el contenido del mensaje, mejorando la percepción y participación pública de la ciencia con los resultados científicos (Bucchi y Saracino, 2016); e involucrar al lector en mayores niveles de pensamiento de información cualitativa y cuantitativa, además de ser un medio persuasivo y un incipiente a la alfabetización visual de los lectores (Lazard y Atkinson, 2015).

En el ámbito de la educación también se han realizado investigaciones que enfatizan la visualización e infografía como herramienta efectiva para potenciar los procesos de comunicación y enseñanza aprendizaje en las aulas de estudio en materias relativas a la salud (Shanks y otros, 2017; Mc Sween-Cadieux y otros, 2018; Ozdal y Ozdamli, 2017; Shafipoor y otros, 2016; Kibar y Akkoyunlu 2014; Clark y Lyons, 2010), las ciencias naturales (Davidson, 2014), las matemáticas (Baglama y otros, 2017) y las ciencias químicas (Mitchell y otros, 2017).

\subsection{Componentes y recursos de expresión de la infografía científica de prensa}

Los elementos de la infografía científica de prensa se encuentran integrados por diversos componentes morfo semánticos, los cuales se dividen en objetos textuales y objetos gráficos. Su configuración determina el cómo se dice el mensaje, empleando mecanismos y técnicas que buscan reforzar y potenciar la comprensión del mensaje. Los objetos textuales se componen de signos de naturaleza arbitraria en su denotación, y tienen como finalidad encabezar el eje general de la información (contexto informativo) a través de un título, subtítulo, sumario y etiquetas de texto vinculadas a los objetos gráficos (encabezados, cajas de texto, rótulos, tablas, listas) (Montes, 2017).

Los objetos gráficos, por su parte, se componen de signos icónicos (figurativo y no figurativo) que tienen como finalidad encabezar el eje específico de la información a través de distintos recursos de expresión, los cuales conllevan a distintos tipos de correspondencia: literal, convencional e híbrida (Engelhardt, 2002). Los objetos gráficos de correspondencia literal presentan un nivel de iconicidad alto, es decir, utilizan estructuras espaciales físicas que muestran una semejanza con el objeto o estructura física que se quiere decir (imágenes icónicas). Por ejemplo, la visualización topográfica de los arrecifes monumentales de Australia; la estructura interna de un objeto o ser vivo; la visualización de la masa de la Tierra a lo largo de las latitudes, y ordenada por vegetación y uso de suelo (Montes, 2017).

Estos objetos utilizan recursos de expresión como:

- Imagen sintética: visualiza y simula eventos o fenómenos científicos a través de imágenes provenientes de las técnicas de visualización científica o imágenes de satélite de alta resolución (Berenguer, 1991). Por ejemplo, la simulación y visualización de que pasaría si la tierra no pudiera girar en su propio eje.

- Ilustración: representa imágenes figurativas (pictóricas) como puede ser la apariencia externa o interna de un objeto o ser vivo con una gran registro y precisión.

- Dibujo esquemático: representa rasgos figurativos esenciales de un objeto o ser vivo, los cuales hacen referencia explícita a un significado.

- Fotografía: documenta, registra, enriquece y da un mayor soporte a la información como hecho ineludible entre fotografía y realidad.

- Caricatura: representa el retrato de un personaje o la escenificación de un acontecimiento exagerando algunos rasgos pictóricos con un toque humorístico (Montes, 2017).

- Mapa raster y mapa vectorial: el primero, se utiliza para representar las características topográficas o uso de suelo de una región a través de imágenes de satélite de alta resolución, y el segundo, para representar gráfica y métricamente una porción de territorio proyectada de manera bidimensional a través de imágenes generadas por vectores (Puebla y Gould, 1994).

Estos recursos de expresión utilizan distintas técnicas de visualización de información que tienen como propósito potenciar el mensaje que portan los gráficos. Estas técnicas son señaladas por Costa (1999) y Montes (2017) como:

- Transparencia: técnica que permite dejar ver lo más destacado que hay adentro de un cuerpo u objeto.

- Simultaneidad: técnica que deja ver de manera paralela distintos elementos visuales que son objeto de comparación.

- Corte transversal: técnica que deja ver la estructura interna de un cuerpo u objeto diseccionado. 
- Acción: técnica que escenifica un proceso físico.

- Lupa: técnica que permite visualizar con detalle un aspecto clave de la imagen.

- Fragmentación: técnica que permite centrar la atención en una parte clave de la imagen.

Los objetos gráficos de correspondencia convencional, por su parte, representan estructuras espaciales conceptuales como los gráficos de datos que relacionan dos o más variables para expresar el comportamiento de un fenómeno. Por ejemplo, los que miden magnitudes físicas escalares (temperatura, masa, densidad, etc.) y vectoriales (velocidad, intensidad luminosa, fuerza, etc.); los que representan datos estadísticos que permiten visualizar la distribución de frecuencias de variables cuantitativas por categorías o subcategorías (gráfico de barras, gráfico circular, etc.), por periodo de tiempo o ciclo (gráfico de líneas, calendario, etc.) y por correlación (gráfico de dispersión) (Yau, 2013). En todos los casos, lo que se muestra parece defender lo que se entiende por convención, no significa por naturaleza, sino por acuerdo (imágenes no icónicas). Por lo tanto, adquieren un tipo de correspondencia arbitraria o convencional (Engelhardt, 2002; Cairo, 2008).

Finalmente, los objetos gráficos que presentan una correspondencia híbrida (literal y convencional de forma simultánea) se debe a que utilizan estructuras espaciales físicas y conceptuales. Por ejemplo, los mapas estadísticos (también denominados mapas de datos) que representan gráficamente dimensiones conceptuales y físicas para expresar el comportamiento de un fenómeno a partir del análisis datos de unidades territoriales políticas y administrativas de un país o, de un conjunto de países. En esta categoría se encuentran el mapa híbrido y el mapa de flujo. El primero, proporciona datos cualitativos y cuantitativos (chorochromatic-proportional symbol maps and chorochromatic-dot maps), y el segundo, representa por medio de flechas (proporcional o no a una cantidad numérica) el movimiento de objetos, animales o personas de un lugar a otro (Alcíbar, 2017). En todos los casos adquieren un tipo de correspondencia híbrida (literal y convencional).

\subsection{Funciones de la imagen en relación con el texto}

Existen numerosos autores que han clasificado las imágenes en relación con los rasgos morfológicos que presentan $y$, sobre todo, en función de la apariencia formal resultante (Lohse y otros, 1994; Ghenghea, 1998; Darian, 2001; Monterde, 2002; Clark y Lyons, 2010). En este sentido, Prieto (2009) señala que la apariencia formal de las imágenes es resultado del papel que desempeñan en la comunicación mediante una serie de criterios funcionales, por lo que su clasificación debe ir de acuerdo con el papel que desempeñan en el marco del texto del que forman parte en un contexto comunicativo dado.

Bajo esa premisa, la taxonomía de las relaciones imagen-texto propuesta por Marsh y Domas (2003), integra las formas en que las imágenes y el texto interactúan a partir de 49 relaciones agrupadas en tres categorías, según la cercanía de la relación conceptual entre imagen y texto: 1) las que expresan poca relación con el texto, 2) las que expresan una relación próxima al texto y 3) las que van más allá del texto. De acuerdo con Prieto (2009), esa taxonomía engloba de manera general la información gráfica desde el punto de vista de su relación con el texto y refleja la actitud e intención del emisor con respecto al público al que se dirige, por lo cual, puede ser utilizada como directriz para identificar la función comunicativa de cualquier tipo de imagen, incluida la infografía de prensa.

La relación particular de la imagen y el texto de la infografía de prensa se conforma a partir de una intención comunicativa donde converge lo funcional y estético, facilitando la memoria, la adquisición de conocimientos y la resolución de problemas, así como la adquisición de destrezas cognitivas (Prieto, 2009).

\section{OBJETIVOS}

El objetivo general de esta investigación es determinar desde el punto de vista descriptivo y cuantitativo, las características y patrones detallados del tratamiento informativo de la infografía científica de la prensa internacional, para emprender de manera eficaz procesos de comunicación y divulgación de los hechos relativos a la Ciencia y Tecnología (СуТ).

El objetivo general se centra en tres objetivos particulares:

1. Examinar los enfoques informativos presentes en la infografía científica de prensa, y comprobar el grado de asociación con los principales planteamientos informativos utilizados en el discurso de la Divulgación Mediática de la Ciencia y Tecnología (DMCyT); como lo son el divulgativo, el periodístico y el divulgativo periodístico (Elías, 2008).

2. Identificar los objetos gráficos y el grado de correspondencia (literal, convencional e híbrida) (Engelhardt, 2002) que adopta la infografía científica de prensa, de acuerdo con los planteamientos informativos que se presenten. 
3. Determinar las funciones comunicativas de la infografía científica de prensa, a través de las principales relaciones imagen-texto (poca relación con el texto, relación próxima al texto y más allá del texto) propuestas por Marsh y Domas (2003).

\section{METODOLOGÍA}

\subsection{Muestra}

Se realizó un análisis de contenido a una muestra de infografías científicas que fueron premiadas en el concurso Malofiej durante el periodo de 2009 a 2019 (el año más reciente de la publicación de los premios al momento de iniciar esta investigación). Este certamen es auspiciado por la SND-E (Society for News Design - capítulo español) y la Universidad de Navarra, España; este tiene como objetivo dar cabida y reconocimiento a trabajos infográficos que han sido publicados en periódicos y revistas de todo el mundo.

Para la selección de la muestra se revisaron las listas de premios oficiales que publica el certamen anualmente; para ello se realizó un trabajo documental (búsqueda, selección y clasificación de gráficos) para identificar las infografías que se encontraban dentro de los cinco grupos temáticos relativos a la CyT de acuerdo con Cortiñas (2010), y que representan los asuntos de mayor recurrencia en la infografía científica de prensa como: 1) la meteorología, el medioambiente y las catástrofes naturales (71 unidades), 2) la biología, la salud y la medicina $(46), 3$ ) la astronomía y los temas espaciales (19), 4) la tecnología y los artefactos tecnológicos (9) y 5) las matemáticas, la química y la física (4). De lo anterior, se obtuvo un universo o población de 149 infografías científicas. Se excluyeron los gráficos hechos por agencias, los correspondientes a la categoría 'portafolios', 'promocionales' y 'especiales'.

\subsection{Instrumento de codificación, recolección y análisis}

Se desarrolló una ficha de codificación como instrumento de recolección de datos, utilizando un enfoque integral inductivo y deductivo con 15 variables como descriptores de las principales propiedades de las piezas infográficas. Las variables se agruparon por afinidad lógica en cuatro categorías y de acuerdo con los siguientes criterios, autores y referencias:

1) Datos biográficos: datos relativos al medio (revista o periódico), idioma, país y fecha de publicación del infográfico.
2) Planteamiento informativo: elementos formales del mensaje periodístico que responden a las preguntas básicas de investigación (Hernando, 1997; Elías, 2008).

- Actores (¿quién?): actores individuales, grupales o instituciones que intervienen como protagonistas del relato informativo.

- Temporalidad (¿cuándo?): temporalidad en la que ha sucedido un acontecimiento o un conjunto de sucesos narrados en el mensaje periodístico.

- Espacio físico (¿dónde?): lugar dónde acontece la acción y puede estar determinada por una región geográfica territorial o un área perteneciente a un cuerpo de un ser vivo u objeto.

- Acción comunicativa (¿qué? y ¿cómo?): acciones o tareas realizadas en el relato informativo que construyen y argumentan el mensaje periodístico como las explicaciones, los planteamientos de posibles escenarios o consecuencias, el análisis de datos estadísticos o la medición de magnitudes físicas escalares (temperatura, masa, densidad, etc.) y vectoriales (velocidad, intensidad luminosa, fuerza, etc.) (Montes, 2017).

- Fuentes de documentación: identifica la procedencia y los tipos de fuentes de documentación.

3) Objetos gráficos: signos icónicos figurativos y no figurativos y el tipo de correspondencia que adoptan (modo de expresión) (Engelhardt, 2002).

- Literal: objetos gráficos figurativos (pictóricos) que utilizan estructuras espaciales físicas que muestran una semejanza con el objeto físico o estructura física que se quiere decir (ilustraciones, dibujos esquemáticos, caricatura, etc.).

- Convencional: objetos gráficos no figurativos (abstractos) que utilizan estructuras espaciales conceptuales para expresar el comportamiento de un fenómeno (gráfico estadístico y gráfico que mide magnitudes físicas escalares y vectoriales).

- Híbrido (literal y convencional): objetos gráficos que utilizan estructuras espaciales físicas y conceptuales de forma simultánea para expresar el estado y comportamiento de un fenómeno (mapa estadístico y mapa de flujo). 
4) Función de los infográficos: Registro de 19 de 46 aplicaciones de las principales relaciones imagen-texto de Marsh y Domas (2003), las cuales se agrupan en tres categorías.

- Poca relación con el texto: variables que definen las imágenes con poca relación con el texto, ya que éstas ejercen una función más orientada a expresar un efecto agradable (estético) en sus elementos, sin que esto necesariamente produzca efectos reales en la comprensión o en la memoria del lector.

- Relación próxima al texto: variables que definen una relación estrecha entre las imágenes y el texto para la interpretación del mensaje, ya que ambos son ejes conductores (didáctico) de significado.

- Más allá del texto: variables que definen el desempeño de las imágenes más allá de lo que el texto puede lograr, proporcionando la representación visual de un concepto o proceso abstracto que son visualizados en forma concreta para facilitar el recuerdo y la aprehensión de un conocimiento (función cognitiva). En esta categoría se integró la aplicación correlación debido a la pertinencia que se observó en los materiales empíricos recopilados de la presente investigación. Esta aplicación se relaciona con un tipo de correlación estadística, es decir, la relación de una variable en función de otra, como: soldados por habitante o gasto en defensa per cápita (Cairo, 2011).

La ficha de codificación se estructuró a partir de cuatro categorías. En la columna izquierda se encuentran los nombres de las variables de cada categoría -y en algunos casos- se citan los autores que fueron tomados de referencia para designar dichas variables. En la columna derecha aparecen los valores de las variables, antecedidos en algunos casos por el símbolo $\bigcirc$ para indicar valores de respuesta de elección única; y el símbolo $\square$ para indicar valores de respuesta con posibilidad de elección múltiple (ver anexo I).

El proceso de codificación se realizó entre el 8 de abril y el 10 de mayo de 2019. Para asegurar la fiabilidad de criterios (intercoder reliability), fue seleccionada una submuestra aleatoria del $50 \%$ de la muestra para el acuerdo entre investigadores. El valor medio de acuerdo con las variables que fueron consideradas fue de .80; para la codificación y captura de los registros de variables y descriptores de las propiedades de las piezas infográficas, se utilizó como herramienta de apoyo el software Atlas.ti (versión 1.5.0).

\section{RESULTADOS}

\subsection{Categoría 1: Datos biográficos}

Los resultados en esta categoría se concretan en beneficio de la economía de espacio a través de la variable Medio (periódico o revista), considerada la más relevante de esta categoría.

\section{Medio}

La muestra de infografías científicas de prensa objeto de análisis, registra 34 medios de todo el mundo (revistas y periódicos) que han sido premiados en el concurso Malofiej durante el periodo de 2009 a 2019 (10 ediciones del concurso); estos trabajos representan las mejores prácticas para emprender de manera eficaz procesos de comunicación y divulgación de la CyT dentro de los cinco grupos temáticos establecidos en esta investigación. Los medios de publicación más premiados en orden decreciente por número de unidades fueron los que se muestran en la figura 1.

La revista National Geographic y el periódico The New York Times se coronan como los medios estadounidenses en formato impreso con mayor reconocimiento en esos campos temáticos. A esos medios les continúan con un mismo número de infografías premiadas los periódicos South China Morning Post (China) y Clarín (Argentina). Después, se sitúan los periódicos El Telégrafo (Ecuador), Die Zeit (Alemania) y La Vanguardia (España). El resto de los medios se han unificado y presentado como Otros, ya que cuentan con un número menor de 4 unidades como premios en esos campos temáticos.

\subsection{Categoría 2: Planteamiento informativo}

A partir de la codificación y cuantificación de las variables establecidas se identificaron tres distintos planteamientos informativos en el total de la muestra $(N=149)$. Para ello, tres variables clave fueron tomadas como referencia para identificar y determinar diferencias en el enfoque informativo (fuentes de documentación, temporalidad y acción comunicativa) de acuerdo con Elías (2008). Se identificaron 72 unidades que presentan un grado de asociación con el planteamiento informativo divulgativo; 14 unidades con el planteamiento informativo periodístico; y 63 unidades con el planteamiento divulgativo periodístico.

La tabla I muestra el porcentaje general de distribución de las variables codificadas y sus indicadores descriptivos (elementos formales del mensaje periodístico) que determinan las cualidades y características de los planteamientos informativos presentes en el total de las piezas infográficas. 
Figura 1. Medio de publicación (periódicos y revistas) por número de infografías premiadas

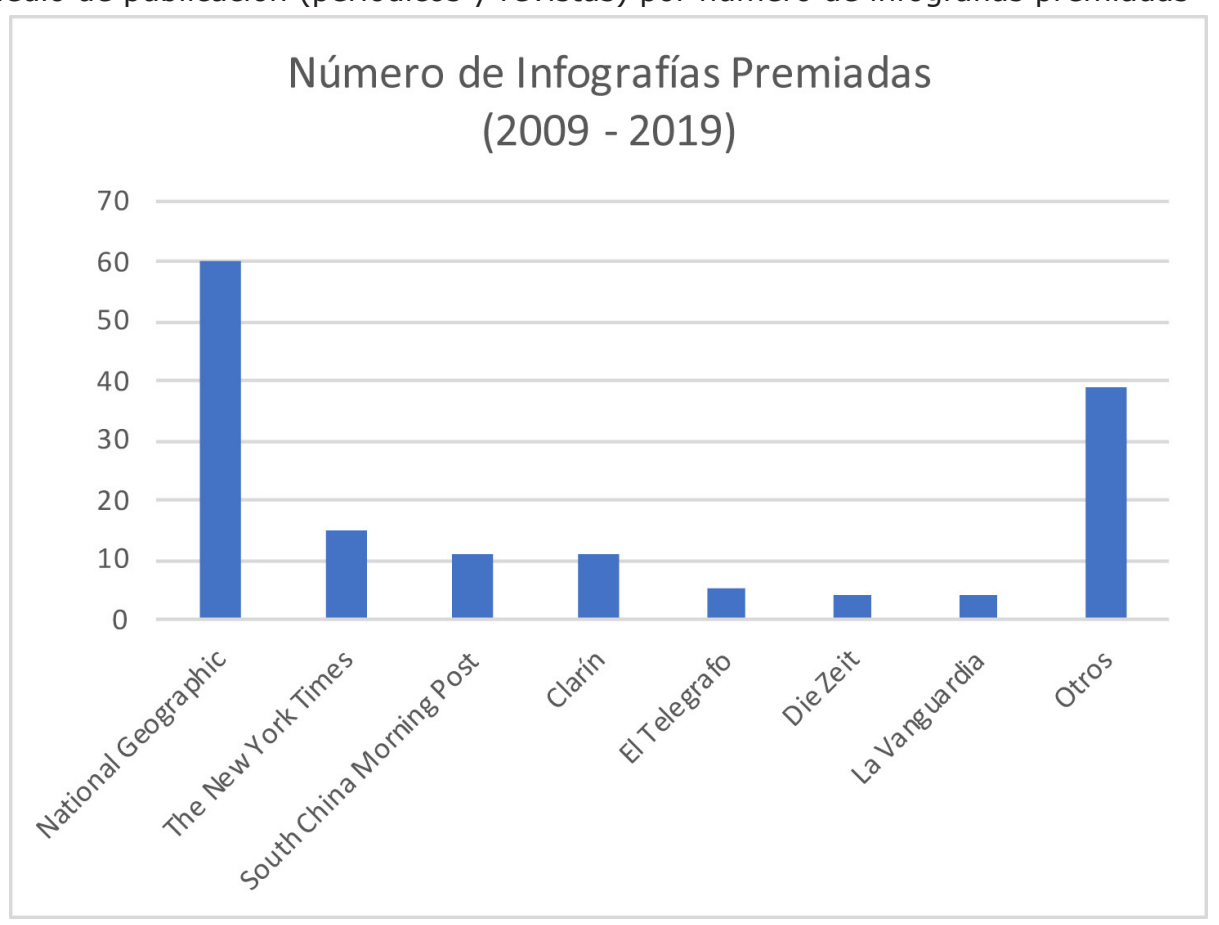

\section{Planteamiento divulgativo}

El planteamiento divulgativo de las piezas infográficas, No indican actores (28\%) concretos, ya que su enfoque se centra en el hallazgo científico. En todo caso, cuando el relato informativo identifica a alguien en concreto, se refiere a una Especie o ser vivo (20\%). Asimismo, el hallazgo es narrado de forma Intemporal (32\%) o remitido al Pasado (16\%) y se centra en Explicaciones causales que implican procesos (31\%) o Explicaciones causales que describen propiedades (17\%); en algunos casos concretos, estas explicaciones van más allá y Miden magnitudes físicas escalares y vectoriales (7\%) o Plantean posibles escenarios o consecuencias $(5 \%)$, basados en supuestos teóricos o hipotéticos que son simulados para exponer casos concretos. Las fuentes de documentación, por su parte, se centran en fuentes directas como: Científico o especialista (25\%); Libros, enciclopedias o manuales especializados, revistas 0 sitios web (13\%); y Universidades, centros de investigación e investigadores y academias de ciencia (11\%) (ver ejemplo en el anexo 2 ).

Estos indicadores muestran un grado de asociación con el planteamiento divulgativo relativo a la ciencia señalado por Elías (2008), el cual se distingue por buscar a la fuente (científicos divulgadores) para construir el discurso centrado en el hallazgo científico y apoyado en la explicación de los fenómenos científicos, narrados de forma intemporal y sin involucrar los intereses de la población o relación alguna con el hecho noticioso.

\section{Planteamiento periodístico}

El planteamiento periodístico de las piezas infográficas se caracteriza por presentar un hecho noticioso relativo a la ciencia donde predominan los actores como Países (7\%), Organismos internacionales pertenecientes al Sistema de las $\mathrm{Na}$ ciones Unidas (2\%) y Dependencias gubernamentales de investigación y servicios (1\%). El hecho noticioso es narrado abarcando distintas temporalidades como el Pasado y presente (5\%); Pasado, presente $y$ futuro $(3 \%)$; Presente y futuro $(1 \%)$; o únicamente el Presente (1\%). El espacio físico, en todos los casos, determina una región geográfica ( $9 \%)$ y presenta una acción comunicativa centrada en el Análisis de datos estadísticos de múltiples variables (28\%); en algunos casos concretos, este análisis va más allá y Plantea posibles escenarios o consecuencias (3\%), fundados en un historial de datos de la ciencia que son utilizados para hacer cálculos y pronósticos. Las fuentes de documentación, por su parte, se centran en fuentes de documentación oficiales como: Organismos internacionales pertenecientes al Sistema de las Naciones Unidas (9\%); Dependencias gubernamentales de investigación y servicios ( $8 \%)$; Universidades, centros de investigación e investigadores y academias de ciencia (3\%); Agencias espaciales (3\%) y Empresas privadas (2\%) (ver ejemplo en el anexo 3 ). 
Tabla I. Distribución general de las variables codificadas que identifican las características de los planteamientos informativos

\begin{tabular}{|c|c|c|c|}
\hline & Divulgativo & Periodístico & $\begin{array}{l}\text { Divulgativo } \\
\text { periodístico }\end{array}$ \\
\hline Variables & $\%$ & $\%$ & $\%$ \\
\hline \multicolumn{4}{|l|}{ Actores } \\
\hline Dependencias gubernamentales de investigación y servicios & $0 \%$ & $1 \%$ & $8 \%$ \\
\hline Agencias espaciales & $0 \%$ & $0 \%$ & $5 \%$ \\
\hline Universidades, centros de investigación e investigadores & $0 \%$ & $0 \%$ & $7 \%$ \\
\hline $\begin{array}{l}\text { Organismos internacionales pertenecientes al sistema de las } \\
\text { Naciones Unidas }\end{array}$ & $0 \%$ & $2 \%$ & $14 \%$ \\
\hline Países & $0 \%$ & $7 \%$ & $8 \%$ \\
\hline Especies o seres vivos & $20 \%$ & $0 \%$ & $0 \%$ \\
\hline No indica actores & $28 \%$ & $0 \%$ & $0 \%$ \\
\hline \multicolumn{4}{|l|}{ Temporalidad } \\
\hline Intemporal & $32 \%$ & $0 \%$ & $0 \%$ \\
\hline Pasado & $16 \%$ & $0 \%$ & $0 \%$ \\
\hline Pasado y presente & $0 \%$ & $5 \%$ & $18 \%$ \\
\hline Presente & $0 \%$ & $1 \%$ & $13 \%$ \\
\hline Presente y futuro & $0 \%$ & $1 \%$ & $4 \%$ \\
\hline Pasado, presente y futuro & $0 \%$ & $3 \%$ & $7 \%$ \\
\hline \multicolumn{4}{|l|}{ Espacio físico } \\
\hline Región geográfica & $23 \%$ & $9 \%$ & $31 \%$ \\
\hline Región perteneciente a un cuerpo & $19 \%$ & $0 \%$ & $11 \%$ \\
\hline No determina espacio físico & $7 \%$ & $0 \%$ & $0 \%$ \\
\hline \multicolumn{4}{|l|}{ Acción comunicativa* } \\
\hline Explicaciones causales que describen propiedades & $17 \%$ & $0 \%$ & $15 \%$ \\
\hline Explicaciones causales que implican procesos & $31 \%$ & $0 \%$ & $27 \%$ \\
\hline Plantea posibles escenarios o consecuencias & $5 \%$ & $3 \%$ & $17 \%$ \\
\hline Analiza datos estadísticos de múltiples variables & $0 \%$ & $28 \%$ & $19 \%$ \\
\hline Miden magnitudes físicas & $7 \%$ & $0 \%$ & $0 \%$ \\
\hline \multicolumn{4}{|l|}{ Fuentes de documentación* } \\
\hline $\begin{array}{l}\text { Universidades, centros de investigación e investigadores y aca- } \\
\text { demias de ciencia }\end{array}$ & $11 \%$ & $3 \%$ & $9 \%$ \\
\hline Científico o especialista & $25 \%$ & $0 \%$ & $2 \%$ \\
\hline $\begin{array}{l}\text { Libros, enciclopedias o manuales especializados, revistas o si- } \\
\text { tios web }\end{array}$ & $13 \%$ & $0 \%$ & $4 \%$ \\
\hline Artículo de revista científica & $5 \%$ & $0 \%$ & $3 \%$ \\
\hline Agencias espaciales & $1 \%$ & $3 \%$ & $9 \%$ \\
\hline Dependencias gubernamentales de investigación y servicios & $0 \%$ & $8 \%$ & $23 \%$ \\
\hline Empresas privadas & $0 \%$ & $2 \%$ & $5 \%$ \\
\hline $\begin{array}{l}\text { Organismos internacionales pertenecientes al sistema de las } \\
\text { Naciones Unidas }\end{array}$ & $0 \%$ & $9 \%$ & $14 \%$ \\
\hline Agencias de noticias & $0 \%$ & $0 \%$ & $1 \%$ \\
\hline
\end{tabular}

\footnotetext{
* Variables de opción múltiple.
} 
Estos indicadores muestran un grado de asociación con el planteamiento periodístico relativo a la ciencia señalado por Elías (2008), donde intervienen diversos actores para analizar y tratar casos que afectan directamente los intereses de la población (hecho noticioso).

\section{Planteamiento divulgativo periodístico}

El planteamiento divulgativo periodístico de las piezas infográficas se caracteriza por presentar ambos enfoques (divulgativo y periodístico). Esto se ve reflejado en el porcentaje de los indicadores descriptivos, los cuales presentan un rango amplio en su distribución, proporcionando una cobertura informativa más amplia en los elementos formales que presenta el mensaje periodístico, así como las fuentes de documentación (ver ejemplo en el anexo 4).

Estos indicadores muestran un grado de asociación con el planteamiento divulgativo periodístico relativo a la ciencia señalado por Elías (2008), el cual se distingue por abordar la explicación de los hallazgos científicos, al tiempo que se contextualiza con criterios periodísticos.

\subsection{Categoría 3: Objetos gráficos}

Los indicadores agrupados en esta categoría informan sobre los recursos de expresión (objetos gráficos) utilizados en cada uno de los grupos de infográficos y el tipo de correspondencia que adoptan. Una misma pieza puede cumplir simultáneamente distintos objetos gráficos y correspondencias, por lo que se da la posibilidad de múltiple elección (ver tabla II).

La distribución de los resultados muestra que los infográficos adoptan objetos gráficos con distintos grados de correspondencia (literal, convencional e híbrida) (Engelhardt, 2002) de acuerdo con el planteamiento informativo que presentan.

Los porcentajes de distribución de los indicadores descriptivos de las piezas infográficas divulgativas reflejan el uso predominante de los objetos gráficos figurativos de correspondencia literal, y prevalece como principal recurso de expresión la ilustración (28\%). Esto quiere decir que los infográficos divulgativos designan más elementos referenciales (imágenes pictóricas) que comparte el científico y los públicos no expertos, frente a lo que sucede con los objetos gráficos de corresponden-

Tabla II. Distribución de las variables codificadas por objetos gráficos y correspondencia de acuerdo con el planteamiento informativo que presentan

\begin{tabular}{|c|c|c|c|}
\hline & Divulgativo & Periodístico & $\begin{array}{l}\text { Divulgativo } \\
\text { periodístico }\end{array}$ \\
\hline Variables & $\%$ & $\%$ & $\%$ \\
\hline \multicolumn{4}{|l|}{ Correspondencia literal* } \\
\hline Ilustración & $28 \%$ & $0 \%$ & $27 \%$ \\
\hline Dibujo esquemático & $4 \%$ & $0 \%$ & $3 \%$ \\
\hline Caricatura & $0 \%$ & $0 \%$ & $0 \%$ \\
\hline Mapa raster & $12 \%$ & $0 \%$ & $2 \%$ \\
\hline Fotografía & $1 \%$ & $0 \%$ & $1 \%$ \\
\hline Mapa vectorial & $5 \%$ & $0 \%$ & $4 \%$ \\
\hline Imagen sintética & $3 \%$ & $0 \%$ & $7 \%$ \\
\hline \multicolumn{4}{|c|}{ Correspondencia convencional o arbitraria* } \\
\hline Gráficos estadísticos & $0 \%$ & $7 \%$ & $25 \%$ \\
\hline Gráficos que miden magnitudes físicas & $7 \%$ & $0 \%$ & $0 \%$ \\
\hline \multicolumn{4}{|c|}{ Correspondencia híbrida (literal y convencional) * } \\
\hline Mapa estadístico & $0 \%$ & $1 \%$ & $0 \%$ \\
\hline Mapa de flujo & $0 \%$ & $1 \%$ & $0 \%$ \\
\hline
\end{tabular}

* Variables de opción múltiple. 
cia convencional. En todo caso, cuando se designan estos últimos, se utilizan gráficos que miden magnitudes físicas escalares y vectoriales (7\%), y se representan de forma simultánea junto con los objetos gráficos de correspondencia literal con el propósito de profundizar en las capas informativas del mensaje (ver ejemplo en el anexo 2).

Los porcentajes de distribución de los indicadores descriptivos de las piezas infográficas periodísticas reflejan el uso predominante de los objetos gráficos no figurativos de correspondencia convencional, y prevalece como principal recurso de expresión el gráfico estadístico (7\%). Esto quiere decir que los infográficos periodísticos se inclinan por designar elementos conceptuales a partir de la representación de imágenes abstractas o arbitrarías que son entendidas por acuerdo o aprendizaje previo por parte del lector. En una escala porcentual inferior, se utilizan los objetos gráficos de correspondencia híbrida como el mapa estadístico ( $1 \%$ ) y el mapa de flujo ( $1 \%$ ) (ver ejemplo en el anexo 3 ).

Por último, los porcentajes de distribución de las piezas infográficas identificadas como divulgativas periodísticas, reflejan un rango amplio de distribución de los objetos gráficos de correspondencia literal y convencional (no híbrida). Esto quiere decir, que los infográficos divulgativos periodísticos mezclan como unidades independientes ambos tipos de objetos para mostrar diversas formas de expresar la información (ver ejemplo en el anexo 4).

\subsection{Categoría 4: Función de los infográficos}

Los indicadores agrupados en esta categoría informan sobre las funciones de los grupos de infográficos de acuerdo con el porcentaje con el que aparecen las principales relaciones imagen-texto. Una misma pieza tiene la posibilidad de cumplir simultáneamente distintas relaciones imagen-texto, por lo que se da la posibilidad de múltiple elección (ver tabla III).

Los porcentajes de distribución de los indicadores descriptivos correspondientes a la variable poca relación con el texto, predominan en todas las piezas infográficas, independientemente del planteamiento informativo que adopten. Esto quiere decir que en todos los casos se ejerce una función estética y, por tanto, competir por la atención del lector.

A partir de la segunda (relación próxima al texto) y tercera variable (más allá del texto) se pueden observar diferencias en la distribución de los porcentajes. Por ejemplo, las piezas infográficas divulgativas tienen un mayor porcentaje de distribución en la variable relación próxima al texto, por lo cual, texto e imagen son por igual ejes conductores de significado con un enfoque didáctico (ver ejemplo en el anexo 2 ).
Las piezas infográficas periodísticas, por su parte, tienen un mayor porcentaje de distribución en la variable más allá del texto, ya que las imágenes proporcionan la representación de un proceso abstracto de forma concreta para facilitar el conocimiento de algo, incrementando la capacidad cognitiva del lector (ver ejemplo en el anexo 3).

Por último, las piezas infográficas divulgativas periodísticas, siguen manteniendo en todas las variables (relaciones imagen-texto) un porcentaje con un rango amplio de distribución. Esto quiere decir, que las relaciones imagen-texto en estas piezas pueden desempeñar de forma simultánea distintas funciones como la estética, la didáctica y la cognitiva (ver ejemplo en el anexo 4).

\section{CONCLUSIONES}

De acuerdo con los resultados obtenidos podemos afirmar que el tratamiento informativo de la infografía científica de la prensa internacional adopta características y patrones detallados para visualizar y comunicar de forma eficaz la ciencia.

En relación con el primer objetivo, se examinaron los enfoques informativos de la infografía científica de prensa y a través de ello se comprobó el grado de asociación con los principales planteamientos informativos utilizados en el discurso de la Divulgación Mediática de la Ciencia y Tecnología (DMCyT): divulgativo, periodístico y divulgativo periodístico (Elías, 2008). No obstante, se observó que el enfoque periodístico se inclina sustancialmente hacia el análisis, representación y visualización de datos relativos a la ciencia. Esta tendencia ha sido estudiada por Cairo (2017), la cual apunta que es consecuencia de la creciente disponibilidad de datos y herramientas digitales que ha llevado a que los profesionales centren su tarea entre el periodismo y los datos; y a una transformación y rol de trabajo de las actuales salas de redacción de periódicos y revistas de élite.

A partir de lo anterior, se determinó la siguiente tipología de infografías científicas de prensa:

1) Infografía de Divulgación de la Ciencia (IDC).

2) Infografía de Periodismo de Datos de la Ciencia (IPDC).

3) Infografía de Divulgación Periodística de la Ciencia y Tecnología (IDPCYT).

En concreto, se observó que la IDC utiliza una estructura informativa sin contexto periodístico y se centra en el desarrollo de las explicaciones causales de forma intemporal, tomando como principales fuentes de documentación las fuentes directas. Por el contrario, se observó que la IPDC utiliza 
Tabla III. Distribución de las variables codificadas por relación imagen-texto

\begin{tabular}{|c|c|c|c|}
\hline & Divulgativo & Periodístico & $\begin{array}{l}\text { Divulgativo } \\
\text { periodístico }\end{array}$ \\
\hline Variables & $\%$ & $\%$ & $\%$ \\
\hline \multicolumn{4}{|l|}{ Poca relación con el texto* } \\
\hline Cambia de ritmo & $40 \%$ & $8 \%$ & $42 \%$ \\
\hline Dirige & $48 \%$ & $9 \%$ & $42 \%$ \\
\hline Ajusta a estilo & $35 \%$ & $9 \%$ & $42 \%$ \\
\hline Suscita emoción & $48 \%$ & $28 \%$ & $42 \%$ \\
\hline Implica & $16 \%$ & $9 \%$ & $42 \%$ \\
\hline Motiva & $48 \%$ & $9 \%$ & $42 \%$ \\
\hline \multicolumn{4}{|l|}{ Relación próxima al texto* } \\
\hline Reitera & $48 \%$ & $0 \%$ & $42 \%$ \\
\hline Explica & $48 \%$ & $0 \%$ & $42 \%$ \\
\hline Traduce & $48 \%$ & $0 \%$ & $42 \%$ \\
\hline Ubica & $48 \%$ & $9 \%$ & $42 \%$ \\
\hline Relaciona & $48 \%$ & $0 \%$ & $42 \%$ \\
\hline Induce perspectiva & $48 \%$ & $9 \%$ & $42 \%$ \\
\hline Define & $48 \%$ & $0 \%$ & $42 \%$ \\
\hline Modela proceso físico & $48 \%$ & $0 \%$ & $42 \%$ \\
\hline \multicolumn{4}{|l|}{ Más allá del texto* } \\
\hline Interpreta & $48 \%$ & $9 \%$ & $42 \%$ \\
\hline Documenta & $7 \%$ & $9 \%$ & $42 \%$ \\
\hline Desarrolla & $18 \%$ & $9 \%$ & $42 \%$ \\
\hline Correlaciona & $0 \%$ & $9 \%$ & $42 \%$ \\
\hline Transforma & $8 \%$ & $9 \%$ & $42 \%$ \\
\hline Modela proceso cognitivo & $7 \%$ & $9 \%$ & $42 \%$ \\
\hline
\end{tabular}

* Variables de opción múltiple.

una estructura informativa periodística comprometida con el análisis e interpretación de datos relativos a la ciencia, tomando como principales fuentes de documentación las fuentes oficiales. Por último, se observó que la IDPCyT utiliza una estructura con una amplia cobertura informativa para abordar desde diferentes perspectivas los hechos noticiosos, pero, además enfatiza desde un enfoque práctico y social el impacto de la CyT.

En cuanto al segundo de los objetivos planteados, se identificaron los objetos gráficos y el grado de correspondencia (literal, convencional e híbrida) que adopta la infografía científica de prensa de acuerdo con el planteamiento informativo que presenta. A partir de ello, se comprobó cuales son los recursos de expresión que se utilizan para potenciar los objetivos comunicativos.
En concreto, se observó que la IDC asigna elementos referenciales (imágenes pictóricas) de correspondencia literal que comparte el científico y los públicos no expertos, fungiendo a su vez, como un medio de traducción de la ciencia. Por el contrario, se observó que la IPDC asigna elementos conceptuales (códigos, relaciones numéricas, flujo de datos, etc.) de correspondencia convencional o arbitraria que exigen un lector informado e interesado con un mayor nivel de atención y capacidad resolutiva. Finalmente, se observó que la IDPCyT asigna por igual, tanto elementos referenciales (Iiteral) y conceptuales (convencionales) como medio para potenciar la amplia cobertura informativa que presenta.

Por último, el tercero de los objetivos planteados, determina las funciones (relaciones imagen-texto) 
de la infografía científica de prensa de acuerdo con el planteamiento informativo que presenta. Los resultados demostraron que todas las piezas infografías desempeñan una función estética. No obstante, de forma global, cada grupo adquirió distintos efectos potenciales sobre el receptor, de acuerdo con su intención comunicativa (planteamiento informativo).

En concreto, se observó que la IDC adoptó de manera predominante la función estética y didáctica; la IPDC adoptó de manera predominante las funciones estética y cognitiva; y la IDPCyT adoptó la función estética, pedagógica y cognitiva de forma simultánea. Esta última, es considerada la más completa en su tipo.

De todo lo dicho, se resume que la prensa internacional hace uso de la infografía científica para dirigirse de forma estratégica a diversos grupos de lectores no especialistas, con distintas necesidades de información, esto se comprueba a partir de las piezas que se han analizado y los distintos planteamientos informativos que reflejan en sus mensajes, los cuales conllevan al uso particular de determinados recursos de expresión (objetos gráficos),

\section{REFERENCIAS}

Alcíbar, M. (2017). Information visualisation as a resource for popularising the technical-biomedical aspects of the last Ebola virus epidemic: The case of the Spanish reference press. Public Understanding of Science, 27(3), 365-381. https://doi. org/10.1177/0963662517702047

Ancker, J. S.; Senathirajah, Y.; Kukafka, R.; Starren, J. B. (2006). Design features of graphs in health risk communication: A systematic review. Journal of the American Medical Informatics Association, 13(6), 608618. https://doi.org/10.1197/jamia.M2115

Baglama, B.; Yucesoy, Y.; Uzunboylu, H.; Ozcan, D. (2017). Can Infographics Facilitate the Learning of Individuals with Mathematical Learning Difficulties?. International Journal of Cognitive Research in Science, Engineering and Education/IJCRSEE, 5(2), 119-127. https://doi.org/10.5937/ijcrsee1702119B

Berenguer, X. (1991). La imatge sintètica com llenguatge. Temes de disseny (5), 89-96. Recuperado de: https:// www.raco.cat/index.php/Temes/article/view/29196

Bucchi, M.; Saracino, B. (2016). Visual science literacy: Images and public understanding of science in the digital age. Science Communication 38(6): 812819. https://doi.org/10.1177/1075547016677833

Cairo, A. (2017). Nerd journalism: How Data and Digital Technology Transformed News Graphics. Tesis doctoral, Universitat Oberta de Catalunya.

Cairo, A. (2011). El arte funcional: infografía y visualización de información. Madrid: Alamut. desempeñando de manera conjunta, diversas funciones comunicativas. En definitiva, estos productos comunicativos son una gran herramienta para potenciar y emprender de manera eficaz procesos de comunicación y divulgación de la СуT.

Las conclusiones generales que se desprenden de este estudio nos hacen pensar que los resultados expuestos en este artículo pueden contribuir al proceso de planificación y diseño de los mensajes relativos a la CyT, ya que proporcionan un marco referencial de soluciones comunicativas para razonar sobre las propuestas más pertinentes que buscan adecuarse a los objetivos comunicativos que se persiguen. No obstante, se debe señalar que estos resultados son reflejo de las actuales normas, tendencias y estrategias comunicativas que subyacen de los diversos relatos de СуT que surgen en revistas y periódicos de élite de referencia internacional y, por lo tanto, no representan una generalidad. Sin embargo, son una referencia mundial y un modelo de buenas prácticas en el quehacer de la divulgación y el periodismo científico en los medios impresos.

Cairo, A. (2008). Infografia 2.0: Visualización interactiva de información en prensa. Madrid: Alamut.

Clark, R.; Lyons, C. (2010). Graphics for learning: Proven guidelines for planning, designing, and evaluating visuals in training materials. San Francisco: John Wiley \& Sons.

Cortiñas, S. (2010). La Contribució de la Infografía al Desenvolupament del Periodisme Científic. Periodística: revista acadèmica (12), 65-81.

Costa, J. (1999). Esquemática. Barcelona: Paidós.

Darian, S. (2001). More than meets the eye. The role of visuals in science textbooks. LSP and professional communication (2001-2008), 1(1).

Davidson, R. (2014). Using Infographics in the Science Classroom. The Science Teacher, 81(3): 34-39.

Dur, B. I. U. (2012). Analysis of data visualizations in daily newspapers in terms of graphic design. ProcediaSocial and Behavioral Sciences, 51, 278-283. https:// doi.org/10.1016/j.sbspro.2012.08.159

Elías, C. (2008). Fundamentos de periodismo científico y divulgación mediática. Madrid: Alianza Editorial.

Engelhardt, Y. (2002). The language of graphics: A framework for the analysis of syntax and meaning in maps, charts and diagrams. Unpublished Ph.D. thesis, Institute for Logic, Language and Computation, University of Amsterdam, The Netherlands.

Galesic, M., y Garcia-Retamero, R. (2011). Communicating consequences of risky behaviors: Life expectancy 
versus risk of disease. Patient education and counseling, 82(1), 30-35. https://doi.org/10.1016/j. pec. 2010.02 .008

García-Retamero, R.; Cokely, E. (2013). Communicating health risks with visual aids. Current Directions in Psychological Science 22(5): 392-399. https://doi. org/10.1177/0963721413491570

Ghenghea, V. A. (1998). The Impact of Effective Diagram Design on LSP Text Understanding. In Proceedings of the 11th European Symposium on LSP Identity and Interface Research, Knowledge and Society, Copenhagen, Copenhagen Business School (pp. 783-793).

Hernando, M. C. (1997). Manual de periodismo científico. Bosch Casa Editorial.

Kibar, P. N., y Akkoyunlu, B. (2014). A new approach to equip students with visual literacy skills: Use of infographics in education. In European Conference on Information Literacy (pp. 456-465). Cham: Springer. https://doi.org/10.1007/978-3-319-14136-7_48

Lazard, A.; Atkinson, L. (2015). Putting environmental infographics center stage: The role of visuals at the elaboration likelihood model's critical point of persuasion. Science Communication 37(1): 6-33. https://doi. org/10.1177/1075547014555997

Lohse, G. L.; Biolsi, K.; Walker, N.; Rueter, H. H. (1994). A classification of visual representations. Communications of the ACM, 37(12), 36-50. https://doi. org/10.1145/198366.198376

Marsh, E.; Domas, M. (2003). A taxonomy of relationships between images and text. Journal of Documentation, 59 (6), 647-672. https://doi. org/10.1108/00220410310506303

Mc Sween-Cadieux, E.; Fillol, A.; Ridde, V.; Dagenais, C. (2018). Letter to the editor in response to "No difference in knowledge obtained from infographic or plain language summary of a Cochrane systematic review: three randomized controlled trials" by Buljan et al.(2017). Journal of Clinical Epidemiology. https:// doi.org/10.1016/j.jclinepi.2018.02.020

Mitchell, D.; Morris, J.; Meredith, J.; Bishop, N. (2017). Chemistry Infographics: Experimenting with Creativity and Information Literacy. En Liberal Arts Strategies for the Chemistry Classroom (pp. 113-131). American Chemical Society. https://doi.org/10.1021/bk-2017-1266.ch007
Monterde, R. A. M. (2002). Relationship and dependency between linguistic and non-linguistic forms of concept representation: a study of texts and addressed to experts and students. LSP and Professional Communication, 2 (2), 31-48.

Montes, M. (2017). El Tratamiento informativo de la infografía científica de prensa. Tesis doctoral, Universitat Pompeu Fabra.

Occa, A.; Suggs, L. (2016). Communicating breast cancer screening with young women: An experimental test of didactic and narrative messages using video and infographics. Journal of Health Communication 21(1). http://dx.doi.org/10.1080/10810730.2015.1018611

Ozdal, H.; Ozdamli, F. (2017). The Effect of Infographics in Mobile Learning: Case Study in Primary School. Journal of Universal Computer Science, 23(12), 1256-1275.

Prieto, J. A. (2009). Traducción e imagen: la información visual en textos especializados. Granada: Tragacanto.

Puebla, J. G.; Gould, M. (1994). SIG: sistemas de información geográfica. Síntesis.

Shafipoor, M.; Sarayloo, R.; Shafipoor, A. (2016). Infographic (information graphic): $A$ tool for increasing the efficiency of teaching and learning processes. International Academic Journal of Innovative Research, 3(2), 1-7. https://iaiest.com/data-cms/articl es/20191107034716pmIAJPL1510004.pdf

Shanks, J. D.; Izumi, B.; Sun, C.; Martin, A.; Byker Shanks, C. (2017). Teaching undergraduate students to Visualize and Communicate Public Health data with infographics. Frontiers in public health, 5, 315. https:// doi.org/10.3389/fpubh.2017.00315

Stone, E. R., Bruine de Bruin, W., Wilkins, A. M., Boker, E. M., y MacDonald Gibson, J. (2017). Designing graphs to communicate risks: Understanding how the choice of graphical format influences decision making. Risk analysis, 37(4), 612-628. https://doi.org/10.1111/ risa. 12660

Ware, C. (2012). Information visualization: perception for design. Elsevier.

Yau, N. (2013). Data points: visualization that means something. John Wiley \& Sons. 


\section{ANEXO}

Anexo 1. Ficha de indicadores para analizar el tratamiento informativo de la infografía científica de prensa

\begin{tabular}{|c|c|}
\hline Categoría 1: Datos & ficos \\
\hline Medio & \\
\hline Idioma & \\
\hline País & \\
\hline Fecha de publicación & \\
\hline Categoría 2: Plante & to informativo \\
\hline $\begin{array}{l}\text { Actores } \\
\text { (Elías, 2008) }\end{array}$ & $\begin{array}{l}\square \text { 1. Dependencias gubernamentales de investigación y servicios } \\
\square \text { 2. Agencias espaciales } \\
\square \text { 3. Universidades, centros de Investigación e investigadores } \\
\square \text { 4. Organismos internacionales pertenecientes al Sistema de las Naciones Unidas } \\
\square \text { 5. Países } \\
\square \text { 6. Especies o seres vivos } \\
\square \text { 7. No indica actores }\end{array}$ \\
\hline $\begin{array}{l}\text { Temporalidad } \\
\text { (Elías, 2008) }\end{array}$ & $\begin{array}{l}\square \text { 1. Intemporal } \\
\square \text { 2. Pasado } \\
\square \text { 3. Pasado y presente } \\
\square \text { 4. Presente } \\
\square \text { 5. Presente y futuro } \\
\square \text { 6. Pasado, presente y futuro }\end{array}$ \\
\hline $\begin{array}{l}\text { Espacio físico } \\
\text { (Elías, 2008) }\end{array}$ & $\begin{array}{l}\square \text { 1. Región geográfica } \\
\square \text { 2. Área que pertenece a un cuerpo (ser vivo u objeto) } \\
\square \text { 3. No determina espacio físico }\end{array}$ \\
\hline $\begin{array}{l}\text { Acción comunicativa } \\
\text { (Montes, 2017) }\end{array}$ & $\begin{array}{l}\square \text { 1. Explicaciones causales que describen propiedades } \\
\square \text { 2. Explicaciones causales que implican procesos } \\
\square \text { 3. Plantea posibles escenarios o consecuencias } \\
\square \text { 4. Analiza datos estadísticos de múltiples variables } \\
\square \text { 5. Mide magnitudes físicas escalares y vectoriales }\end{array}$ \\
\hline $\begin{array}{l}\text { Fuentes de consulta } \\
\text { (Elías, 2008) }\end{array}$ & $\begin{array}{l}\square \text { 1. Universidades, centros de investigación e investigadores y academias de ciencia } \\
\square \text { 2. Científico o especialista } \\
\square \text { 3. Libros, enciclopedias o manuales especializados, revistas o sitios web } \\
\square \text { 4. Artículo de revista científica } \\
\square \text { 5. Agencias espaciales } \\
\square \text { 6. Dependencias gubernamentales de investigación y servicios } \\
\square \text { 7. Empresas privadas } \\
\square \text { 8. Organismos internacionales pertenecientes al Sistema de las Naciones Unidas } \\
\square \text { 9. Agencias de noticias }\end{array}$ \\
\hline
\end{tabular}




\begin{tabular}{|c|c|}
\hline \multicolumn{2}{|c|}{ Categoría 3: Objetos gráficos } \\
\hline $\begin{array}{l}\text { Correspondencia literal } \\
\text { (Engelhardt, 2002) }\end{array}$ & $\begin{array}{l}\square \text { 1. Ilustración } \\
\square \text { 2. Dibujo esquemático } \\
\square \text { 3. Caricatura } \\
\square \text { 4. Mapa raster } \\
\square \text { 5. Fotografía } \\
\square \text { 6. Mapa vectorial } \\
\square \text { 7. Imagen sintética }\end{array}$ \\
\hline $\begin{array}{l}\text { Correspondencia } \\
\text { convencional } \\
\text { (Engelhardt, 2002) }\end{array}$ & $\begin{array}{l}\square \text { 1. Gráficos estadísticos } \\
\square \text { 2. Gráficos que miden magnitudes físicas escalares y vectoriales }\end{array}$ \\
\hline $\begin{array}{l}\text { Correspondencia híbrida } \\
\text { (Engelhardt, 2002) }\end{array}$ & $\begin{array}{l}\square \text { 1. Mapa estadístico } \\
\square \text { 2. Mapa de flujo }\end{array}$ \\
\hline \multicolumn{2}{|c|}{ Categoría 4: Función de los infográficos } \\
\hline $\begin{array}{l}\text { Poca relación con el texto } \\
\text { (Marsh y Domas, 2003) }\end{array}$ & $\begin{array}{l}\square \text { 1. Cambio de ritmo } \\
\square \text { 2. Dirige } \\
\square \text { 3. Ajusta a estilo } \\
\square \text { 4. Suscita emoción } \\
\square \text { 5. Implica } \\
\square \text { 6. Motiva }\end{array}$ \\
\hline $\begin{array}{l}\text { Relación próxima al texto } \\
\text { (Marsh y Domas, 2003) }\end{array}$ & $\begin{array}{l}\square \text { 1. Reitera } \\
\square \text { 2. Explica } \\
\square \text { 3. Traduce } \\
\square \text { 4. Ubica } \\
\square \text { 5. Relaciona } \\
\square \text { 6. Induce perspectiva } \\
\square \text { 7. Define } \\
\square \text { 8. Modela proceso físico }\end{array}$ \\
\hline $\begin{array}{l}\text { Más allá del texto } \\
\text { (Marsh y Domas, 2003) }\end{array}$ & $\begin{array}{l}\square \text { 1. Interpreta } \\
\square \text { 2. Documenta } \\
\square \text { 3. Desarrolla } \\
\square \text { 4. Correlaciona } \\
\square \text { 5. Transforma } \\
\square \text { 6. Modela proceso cognitivo }\end{array}$ \\
\hline
\end{tabular}


Anexo 2. Infográfico de divulgación de la ciencia (Premio Oro Malofiej 21), que explica de forma ilustrada la mecánica del cuerpo y la física del pingüino que ayuda a que se dispare al momento de salir del agua

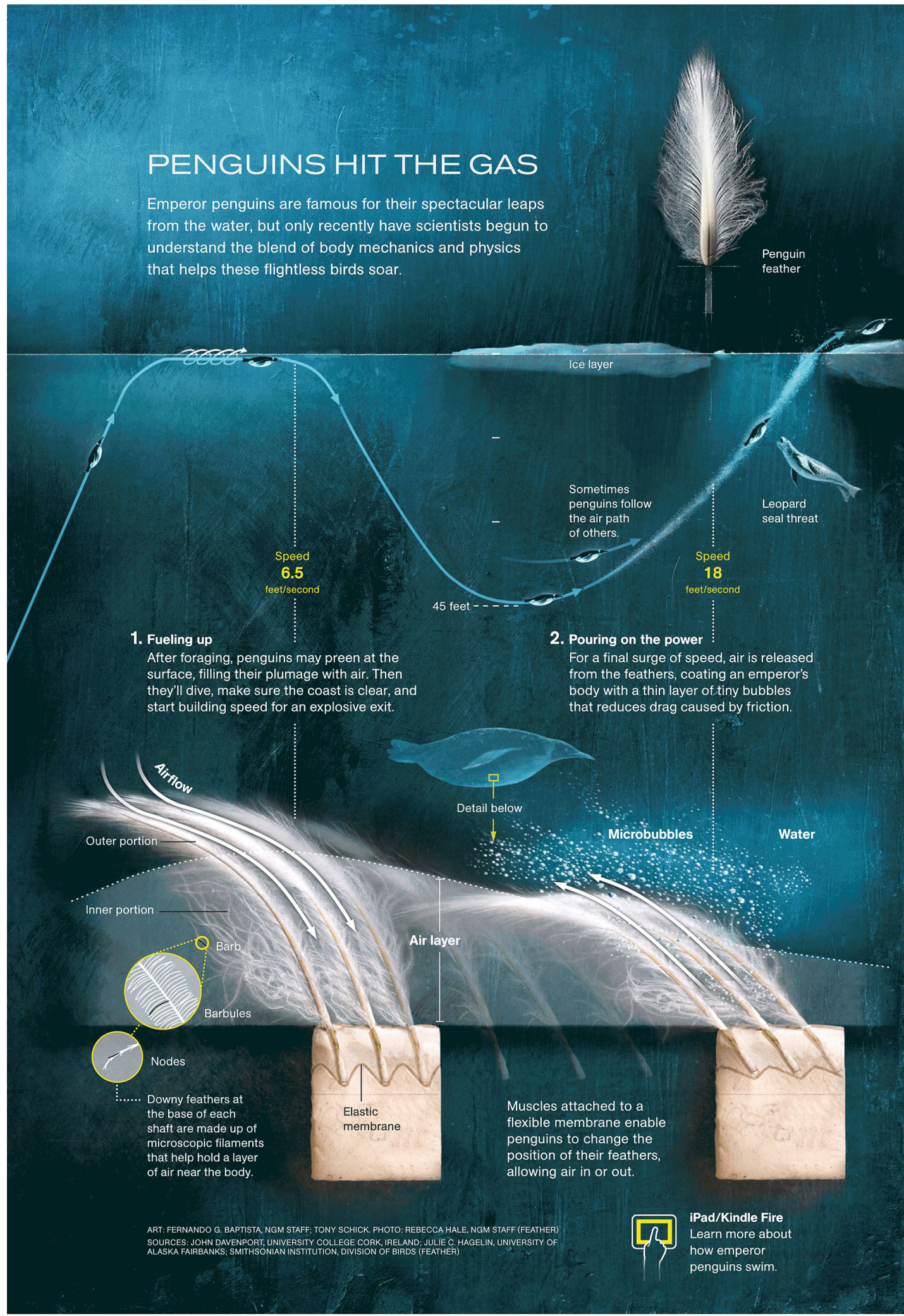

Fuente: revista National Geographic, volumen 222, no 5. Noviembre, 2012. Estados Unidos. 
Anexo 3. Infográfico periodístico de la ciencia (Premio Bronce Malofiej 21) que analiza y visualiza datos sobre el número de objetos espaciales (escombros) que se han incrementado desde la década de los 50s, a causa de las pruebas espaciales y accidentes ocurridos entre satélites de distintos países; y las medidas de limpieza que están contemplando los gobiernos involucrados

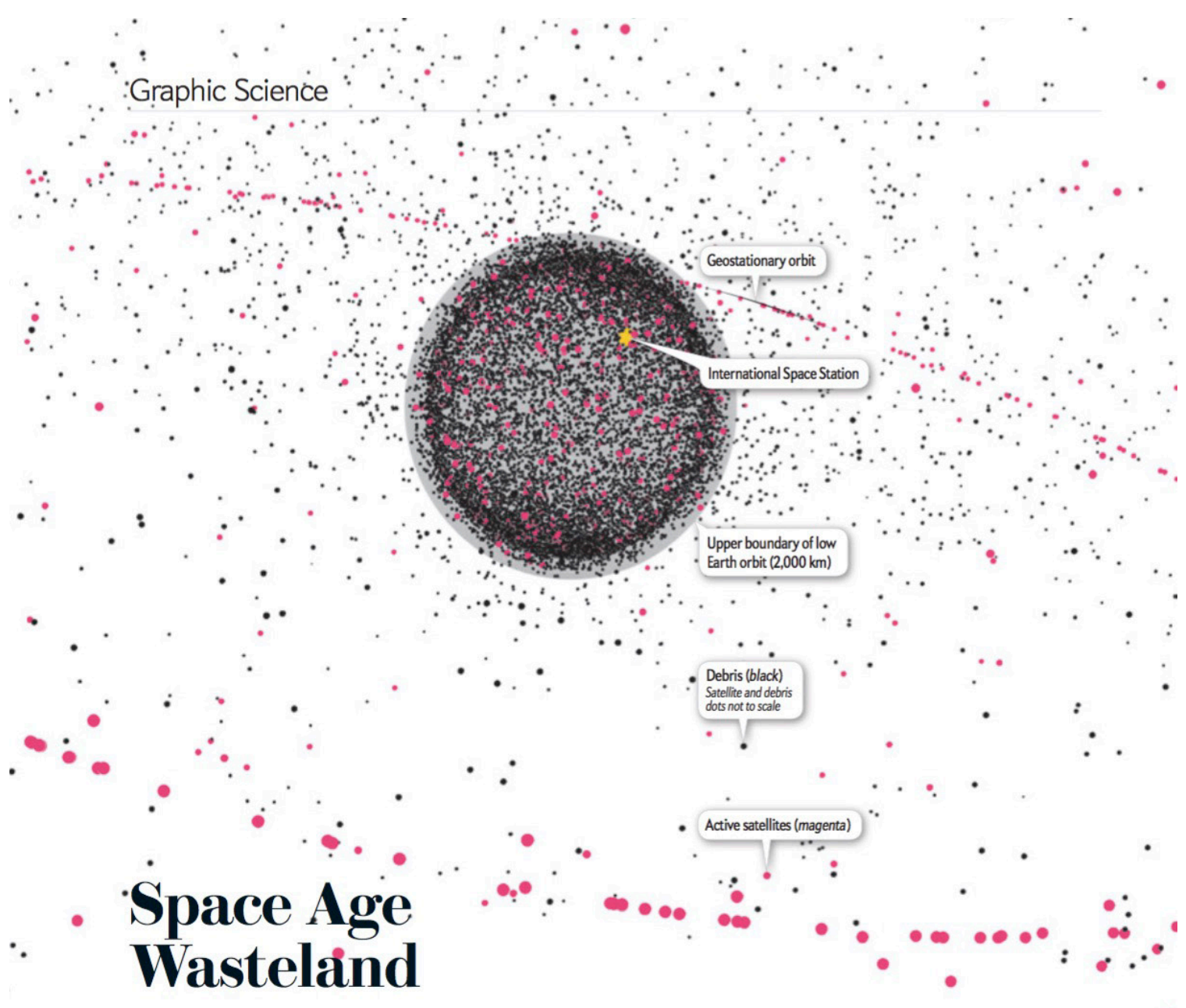

\section{Debris in orbit is here to stay}

Space may be incomprehensibly vast, but Earth's environs are crowded with junk. Spent rockets, derelict spacecraft, satellite fragments and loose hardware now form a cloud of debris that poses a threat to orbiting satellites and astronauts. Sky watchers have catalogued more than 16,000 objects larger than about 10 centimeters, most of them in low Earth orbit, at altitudes of 200 to 2,000 kilometers (right).

And the junk is self-sustaining. If humankind were to cease all spacefaring activities, the hardware we have already cast off would continue to collide and fragment into bits for centuries. Maintaining current launch rates would make the problem even worse. The number of space objects has shot up in the past five years because of China's 2007 test of an antisatellite weapon and the 2009 crash between Russian and U.S. satellites. Governments are contemplating cleanup measures but have yet to devise a workable solution. -John Matson

\section{SCIENTIFIC AMERICAN ONLINE}

See more data in an interactive graphic at ScientificAmerican.com/apr2012/graphic-science

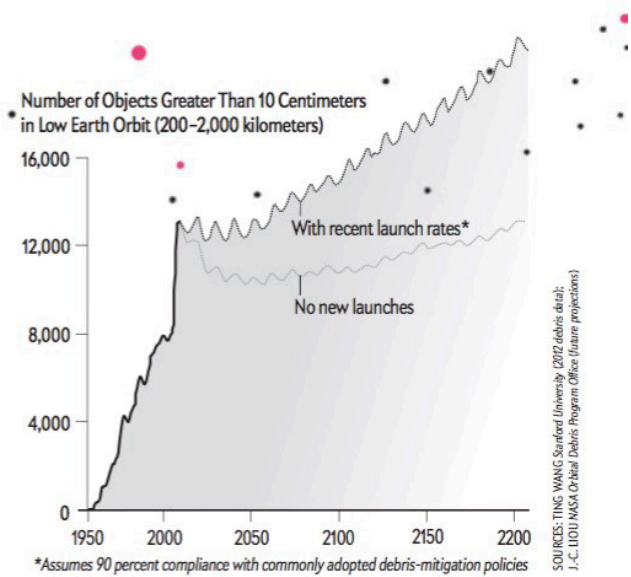

Graphic by Jan Willem Tulp

88 Scientific American, April 2012

(c) 2012 Scientific American

Fuente: revista Scientific American, volumen 306, No 4. Abril, 2012. Estados Unidos. 
Anexo 4. Infográfico de divulgación periodística (Premio Bronce Malofiej 22) que explica las causas del brote de algas verdes en las aguas del mar Oriental de Shandong (China), originados por factores humanos, y la amenaza que representa para la vida marina. Además, visualiza la zona afectada del año 2008 en comparación con la del 2013, y las zonas afectadas en el mundo.

\section{The dead zone}

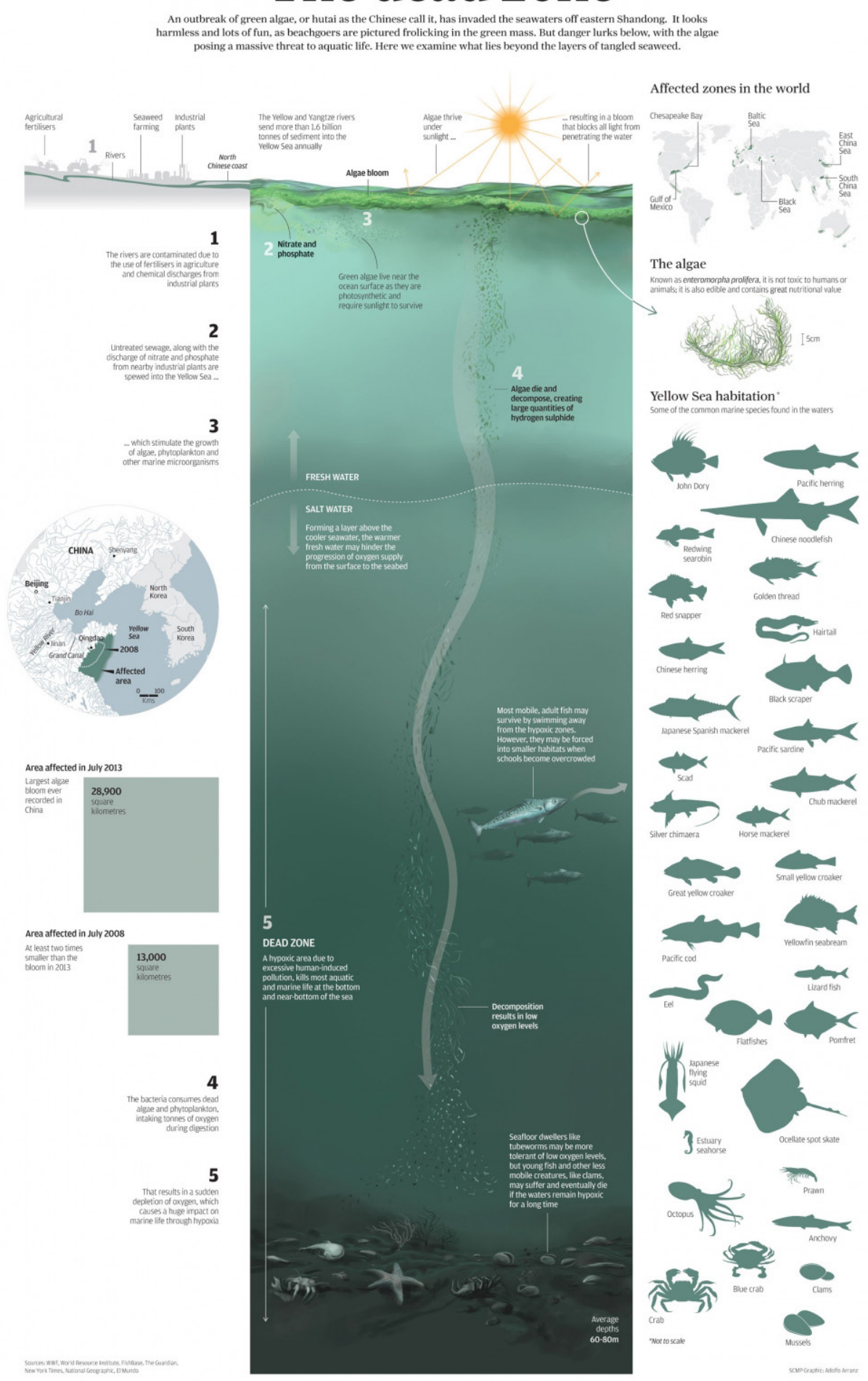

Fuente: periódico South China Morning Post. Julio, 2013. Hong Kong, China. 